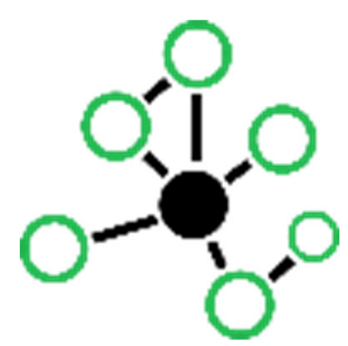

\title{
Advancing interoperable soil data exchange for global soil data information systems
}

\author{
Van Egmond, F. ${ }^{1}$, B. Kempen ${ }^{1}$, N. Batjes ${ }^{1}$, L. de Sousa ${ }^{1}$ \\ ${ }^{1}$ International Soil Reference and Information Centre, Wageningen University \& Research, Droevendaalsesteeg 3, \\ $6708 P B$ Wageningen, the Netherlands \\ Corresponding author's e-mail: fenny.vanegmond@wur.nl
}

In order to be able to address local, regional and global issues such as sustainable land management, food security, climate change mitigation and soil-related indicators of the UN Agenda for Sustainable Development the need for reliable, relevant and accurate soil information and data is increasing. Currently, the soil data community is providing an increasing amount of open soil data through conventional data download options and as webservices. Some of these portals can be classified as FAIR, some follow OGC standards for geographical data, some are standardised to a common (metadata) format like INSPIRE or ISO but a lot of data is still stored in silos. The data is hosted and collected by multiple organisations, structured in different ways and provided in different formats, making it difficult to share, standardise and combine data.

At present, ISRICs information infrastructure is one of the few that serves standardised point soil data from around the globe, from various data providers with assigned data licenses in a findable, accessible, interoperable and reusable way. We follow OGC standards (wfs, wms, wcs) and use GeoNetwork, GeoServer and REST to serve point soil data from WoSIS (ISRICs World Soil Information System) and global soil property and soil type maps derived from that (Soilgrids).

Currently, the Global Soil Partnership aims to develop, build and implement the distributed Global Soil Information System (GLOSIS) and has appointed ISRIC to host the GSP Soil Data Facility. The specifications of this distributed system that empowers data owners and needs to be flexible in the required data informatics capacity are now being written and discussed. In 2019 some pilots will start to test the different SIS options. One is to store data in a standardised support node, one is to use a standard template to setup a local CountrySIS and one is to connect an existing CountrySIS to GloSIS using a common soil data exchange format.

This common soil data exchange format will be developed by GSP pillar 5 based on the previous work in ISO, OGC and INSPIRE. To complement this institutional process the community has initiated a Soil Data WG within GODAN (Global Open Data for Agriculture and Nutrition) and one within the international soil science community (IUSS) which are open to anyone, from private to public sector, who has an interest in supporting alignment on a global soil data exchange format for open soil data such as is available in a number of other domains already. This WG aims to promote the development and the use of interoperable exchange formats by developing use cases and supporting capacity building as well as building high level advocacy.

The overall aim of both GSP' and ISRIC's information products is to be able to provide FAIR Linked Open Soil Data for regional and global studies without spending half of the budget on data preparation and harmonisation within each project. 